Edición Extraordinaria. p.p. 148 - 156

Memorias del VIII Encuentro Nacional de Experiencias en Enseñanza de la Biología y la Educación Ambiental. III Congreso Nacional de Investigación en Enseñanza de la Biología.

\title{
Conocimientos faunísticos de estudiantes de grado sexto de una escuela rural del municipio de Fómeque, Cundinamarca
}

\author{
Rubinsten Hernández Barbosa ${ }^{1}$ \\ Claudia Patricia Neusa Vargas ${ }^{2}$
}

\section{Resumen}

En este texto se describe una experiencia investigativa que tuvo por objetivo caracterizar el conocimiento que los estudiantes de grado sexto de una escuela rural del municipio de Fómeque, Cundinamarca, tienen sobre la fauna de su región. La investigación se desarrolló con 20 estudiantes de grado sexto de la Institución Educativa Rural San Lorenzo, adscrita al Municipio de Fómeque, Cundinamarca. Los resultados indican que el conocimiento que tienen los estudiantes sobre los animales que conocen se puede organizar en cinco categorías, cuatro de ellas dentro del conocimiento ecológico. Los resultados de este trabajo invitan a repensar y reconfigurar modelos alternativos de enseñanza de las ciencias más incluyentes y contextuales.

Palabras clave: contexto cultural, currículo, educación en ciencias naturales, fauna.

\section{Introducción}

B

I

0

$P$

0

N

E

$N$

C

I

A

En este texto se describe parte de la experiencia investigativa ${ }^{3}$ que tuvo por objetivo explorar los conocimientos faunísticos que los estudiantes de grado sexto de la Institución Educativa Rural Departamental San Lorenzo tienen sobre la fauna de la región. El interés surge por identificar, reconocer y valorar dichos conocimientos en poblaciones no marcadas étnicamente, y que son valiosos a la hora de incluirlos en los currículos institucionales, con el ánimo de incorporarlos en el escenario educativo, más concretamente en las dinámicas de la clase de ciencias naturales.

Lo anterior en consonancia con el reconocimiento de nuevos marcos conceptuales y referenciales más incluyentes y contextuales, que se vienen dando desde hace unas décadas en la enseñanza de las ciencias. En ese sentido, es necesario comprender las relaciones entre la cultura y la educación, donde el contexto cultural juega un papel

1. Docente investigador Universidad Autónoma de Colombia. rhbjd@hotmail.com

2. Licenciada en Química. Universidad Distrital Francisco José de Caldas. Docente Institución Educativa Rural San Lorenzo, Fómeque, Cundinamarca. patricia.neusa@gmail.com

${ }^{3}$. La experiencia que se describe en este artículo forma parte del proyecto de investigación doctoral que actualmente adelanta uno de los autores en el marco de su tesis doctoral titulada El contexto cultural en las prácticas educativas de profesores de ciencias del sector rural: perspectivas para el modelo de formación por cambio didáctico, en el Doctorado Interinstitucional en Educación, Universidad Distrital Francisco José de Caldas. 
Memorias del VIII Encuentro Nacional de Experiencias en Enseñanza de la Biología y la Educación Ambiental. III Congreso Nacional de Investigación en Enseñanza de la Biología.

central, pues es través de éste que los individuos expresan y ponen en evidencia sus formas de relación, intereses, necesidades y saberes, entre otros aspectos. Este reconocimiento conlleva a reconfigurar el papel de la escuela, y dejar de verlo como un espacio unificador, igualitario, neutro, universal y extraterritorial, producto de una ideología de la modernidad, donde los medios de comunicación han tenido y siguen teniendo un gran poder (Bolívar, 2004).

En ese orden de ideas, es fundamental comprender que la educación en general debe interpretarse en un contexto particular, y que la apropiación y construcción de los diferentes tipos de conocimiento depende, entre otros aspectos, de los valores, creencias, intereses y necesidades de las personas (Molina, López y Mojica, 2005). Interpretando lo anterior y ampliando el panorama conceptual que sustenta la experiencia que se describe en este artículo, en el marco de la enseñanza de las ciencias naturales, se pueden identificar cuatro perspectivas (Molina, Martínez, Mosquera y Mojica, 2009).

$\checkmark$ Los universalistas: consideran que el cuerpo de conocimiento y actividad de la ciencia tienen un carácter universal, y por ende su enseñanza debe darse con esta mirada, sin tener en cuenta perspectivas multiculturales.

$\checkmark$ Los multiculturalistas: reconocen otros tipos de conocimientos y plantean el Traditional, Ecological knowledge (TEK) en la enseñanza de las ciencias.

$\checkmark$ Los pluralistas epistemológicos: anotan que el conocimiento científico es solo una forma de conocimiento y señalan la existencia e importancia de otros tipos de conocimientos.

$\checkmark$ Los interculturalistas: resaltan la importancia que tienen para la enseñanza de las ciencias los conocimientos ancestrales y tradicionales. En ese orden de ideas, Jedege (1995) citado por Molina y otros, anota que en la enseñanza de las ciencias es importante considerar dos contextos culturales: el que tiene que ver con la cultura de los estudiantes, y el otro, el contexto cultural de la ciencia occidental

En Colombia, en la enseñanza de las ciencias, ha imperado la perspectiva universalista, lo que ha llevado, por parte del aparato educativo, y de manera específica la escuela, a no considerar, incluso invisibilizar, los conocimientos tradicionales y ancestrales, entre otros, de diferentes grupos humanos. En ese orden de ideas, en la enseñanza de las ciencias naturales es preciso considerar enfoques culturales, donde se reconozca el contexto y la diversidad cultural como factor sustancial al momento de establecer los objetivos, los contenidos y las estrategias de enseñanza, ente otros aspectos (Molina \& Uteges, 2011). Lo anterior en concordancia con lo que declara la Constitución de 1991, donde se reconoce que Colombia es un país pluriétnico, pluricultural y megadiverso. Para 
Memorias del VIII Encuentro Nacional de Experiencias en Enseñanza de la Biología y la Educación Ambiental. III Congreso Nacional de Investigación en Enseñanza de la Biología.

ello es esencial comprender al ser humano como un sujeto que está inmerso en condiciones determinadas que forman su particularidad, y que se desarrolla en espacios y tiempos específicos.

Una comprensión de los aspectos antes mencionados, conlleva necesariamente, a repensar y reconfigurar, por parte del docente de ciencias naturales, aspectos relacionados con la pluriculturalidad, la interculturalidad, el contexto y la diversidad cultural. Para ello, la atención a la diversidad significa un giro en la manera de abordar los procesos de enseñanza, de aprendizaje, de evaluación y de currículo en ciencias. Es bajo esta perspectiva que se desarrolló el trabajo que continuación se describe.

\section{Metodología}

Este proyecto se abordó desde el enfoque de investigación cualitativa, la cual se caracteriza por ser inductiva, parte de los datos para construir cuerpos y referentes teóricos, analiza a los sujetos en sus escenarios naturales desde una perspectiva holística, considerando el contexto sociocultural en el que se encuentran e interactúan (Quenceno y Castaño, 2002). Para McMillan y Schumacher (2010), la investigación cualitativa describe y analiza las opiniones, los pensamientos, las percepciones y las conductas sociales individuales y colectivas de quienes conforman el fenómeno de interés investigativo.

Según Vasilachis (2006), la investigación cualitativa tiene como meta la comprensión de las realidades sociales. Por su parte Maxwell (1998) considera que este tipo de investigación se caracteriza por ser inductiva y hermenéutica, ya que focaliza su interés en el significado y la interpretación, el cual depende del contexto. Teniendo en cuenta que el desarrollo del proyecto se sustentó desde el enfoque investigativo cualitativo, es importante tener presente lo que plantea Geertz (1989) al decir que un objetivo fundamental de este tipo de investigación es aproximarse al universo del otro, ese otro que para este caso son los niños y niñas de la Institución Educativa Rural Departamental San Lorenzo, entre los 11 y 14 años

La institución está adscrita al Municipio de Fómeque, que se ubica sobre la Cordillera Oriental, al sureste del Departamento de Cundinamarca. Es una zona de gran diversidad biológica y riqueza hídrica, pues de las 76.452 hectáreas del Parque Natural Chingaza, 25.882 están en jurisdicción de este municipio. A continuación se especifican los momentos y actividades que se desarrollaron en esta experiencia investigativa.

A. Identificación de la fauna de la región. A los estudiantes se les solicita indicar los animales de la región que ellos conocen. 
Memorias del VIII Encuentro Nacional de Experiencias en Enseñanza de la Biología y la Educación Ambiental. III Congreso Nacional de Investigación en Enseñanza de la Biología.

B. Selección de un animal y caracterización del mismo. De los animales señalados cada uno de los estudiantes debía seleccionar uno y diligenciar un formato en el que diera respuesta a tres preguntas.

- ¿Por qué escogiste ese animal?

- ¿Qué sabes sobre el animal que escogiste?

- ¿Por qué crees que el animal es importante en la naturaleza?

C. Exposición. Los niños y niñas hacen un dibujo del animal que ellos seleccionaron, se les solicita que representen el hábitat natural, que exponen luego en la cartelera del salón.

D. Socialización del trabajo. En esta etapa cada uno de los niños y niñas presenta al grupo su trabajo.

\section{Resultados y discusión}

Considerando las tres etapas mencionadas anteriormente, a continuación se presentan los resultados y se hacen algunos análisis de los mismos a la luz de los objetivos de la actividad y de los marcos conceptuales que sustentan la experiencia. En primer lugar hay que decir que al preguntar por los animales de la región, se destaca la gran diversidad de éstos, tal y como se muestra en la Tabla No 1. Los niños propusieron esta clasificación, considerando criterios como la cercanía al ser humano, que se pueden criar en las fincas, el uso del mismo como fuente de alimento y de compañía.

Se destaca los nombres populares o comunes de algunos de ellos, particularmente llama la atención el de algunas serpientes como la sapa, la cuatro narices, la cacica, la voladora, la coral, la taguaya, que fueron mencionadas especialmente por los niños. También se presentaron diferencias entre los animales que señalan los niños y los que mencionan las niñas. Al revisar la lista de animales en conjunto, se nota que no mencionan invertebrados, y que hay animales de todos los grupos de vertebrados, destacándose el grupo de aves, como también señalan, aunque no lo han visto directamente, el oso de anteojos, animal emblemático del Parque Natural Chingaza.

Tabla No. 1. Animales de la región de Fómeque

\begin{tabular}{|l|l|}
\hline $\begin{array}{l}\text { a. Animales } \\
\text { domésticos }\end{array}$ & $\begin{array}{l}\text { Vaca, perro, gato, burro, caballo, marrano, gallinas, piscos, pavos, } \\
\text { gallos de pelea, conejos, patos, gansos, toros, cabros, chivas, } \\
\text { mulas, loro, pericos, canarios, trucha arcoíris, guabina, mojarra, } \\
\text { corronchos, lisitos. }\end{array}$ \\
\hline b. Animales & Ardilla, armadillo, zorros, comadreja, runchos, lapa, culebras, la \\
\hline
\end{tabular}


Edición Extraordinaria. p.p. 148 - 156

Memorias del VIII Encuentro Nacional de Experiencias en Enseñanza de la Biología y la Educación Ambiental. III Congreso Nacional de Investigación en Enseñanza de la Biología.

\begin{tabular}{|l|l|}
\hline salvajes & $\begin{array}{l}\text { sapa, la cuatro narices, la cacica, la voladora, la coral, la taguaya, } \\
\text { terciopelo, chulos, águila, gavilán, carpintero, cóndor de los Andes, } \\
\text { copetón, tintín, mirla, caicas, garzas, pichonas, perdices, colibrí, } \\
\text { toche, jirivuelos, piparos, el tres pesos, el girasol, verrionas, } \\
\text { azulejos, torcazas, golondrinas, oso de anteojos y venado. }\end{array}$ \\
\hline
\end{tabular}

En cuanto a la selección y caracterización de un animal, en la Tabla No 2, se señalan algunos ejemplos de los animales seleccionados. Frente a la primera pregunta, al analizar los textos de todos los estudiantes, se establece que la selección del animal está sujeta a varios factores, que tienen que ver con los sentimientos que el animal le genera, también con las características físicas, particularmente de color, lo cual está unido al concepto de belleza. También algunos se refirieron al movimiento y al papel que juega en la cadena alimenticia.

Tabla No 2. Caracterización de la fauna de la región

\begin{tabular}{|c|c|c|c|}
\hline $\begin{array}{c}\text { Animal } \\
\text { seleccionado }\end{array}$ & $\begin{array}{l}\text { ¿Por qué escogiste } \\
\text { este animal? }\end{array}$ & $\begin{array}{c}\text { ¿Qué sabes sobre } \\
\text { el animal que } \\
\text { escogiste? }\end{array}$ & $\begin{array}{c}\text { ¿Por qué crees que } \\
\text { el animal es } \\
\text { importante en la } \\
\text { naturaleza? }\end{array}$ \\
\hline Oso de anteojos & $\begin{array}{l}\text { Lo escogí porque } \\
\text { tiene mis dos colores } \\
\text { favoritos, blanco y } \\
\text { negro y a mi } \\
\text { hermanita le gusta. }\end{array}$ & $\begin{array}{lr}\text { Vive en el } & \text { parque } \\
\text { Chingaza, } & \text { tiene } \\
\text { manchas } & \\
\text { amarillentas } & 0 \\
\text { blancas y } & \end{array}$ & $\begin{array}{l}\text { Porque hace parte de } \\
\text { la cadena alimenticia } \\
\text { y está en extinción. }\end{array}$ \\
\hline La sapa (serpiente) & $\begin{array}{l}\text { Porque es larga y de } \\
\text { color café. }\end{array}$ & $\begin{array}{l}\text { Es larga, de color } \\
\text { café, vive entre el } \\
\text { chiquero y las } \\
\text { piedras, caza ratones } \\
\text { y sapos. }\end{array}$ & $\begin{array}{l}\text { Es importante en la } \\
\text { naturaleza porque es } \\
\text { de la cadena } \\
\text { alimenticia y cuando } \\
\text { hay mosco o algo ella } \\
\text { se los come. }\end{array}$ \\
\hline Jirivuelo (Ave) & $\begin{array}{l}\text { Lo escogí porque } \\
\text { conozco donde vive, } \\
\text { porque le quitan las } \\
\text { garrapatas al ganado } \\
\text { y se la pasa con los } \\
\text { pájaros. }\end{array}$ & $\begin{array}{l}\text { Este animal es de } \\
\text { color negro de } \\
\text { tamaño pequeño } \\
\text { vuela por los árboles, } \\
\text { ellos viven en nidos } \\
\text { de hojas y chiquero, } \\
\text { se alimenta de las } \\
\text { garrapatas de los } \\
\text { animales. }\end{array}$ & $\begin{array}{lr}\text { Yo creo que es } \\
\text { importante porque se } \\
\text { reproduce y nacen } \\
\text { muchos más para } \\
\text { comerse } \\
\text { garrapatas. }\end{array}$ \\
\hline Armadillo & $\begin{array}{l}\text { Escogí este animal } \\
\text { porque me llama la } \\
\text { atención, porque es } \\
\text { un animal muy ágil y } \\
\text { es muy rico para } \\
\text { comer. }\end{array}$ & $\begin{array}{l}\text { Lo que yo sé sobre } \\
\text { este animal que } \\
\text { escogí, es que tiene } \\
\text { un caparazón que le } \\
\text { cubre del agua, } \\
\text { también que tiene } \\
\text { una cabeza muy } \\
\text { pequeña, es como de } \\
\text { color gris y café, }\end{array}$ & $\begin{array}{l}\text { Creo que el armadillo } \\
\text { es importante en la } \\
\text { naturaleza porque es } \\
\text { un ser vivo y todo ser } \\
\text { vivo tiene derecho de } \\
\text { pertenecer en la } \\
\text { naturaleza, en un } \\
\text { lugar sano y lleno de } \\
\text { vida. }\end{array}$ \\
\hline
\end{tabular}


Memorias del VIII Encuentro Nacional de Experiencias en Enseñanza de la Biología y la Educación Ambiental. III Congreso Nacional de Investigación en Enseñanza de la Biología.

\begin{tabular}{|l|l|l|l|}
\hline & & $\begin{array}{l}\text { corre velozmente, } \\
\text { vive en el monte en } \\
\text { cuevas muy grandes. }\end{array}$ & \\
\hline $\begin{array}{l}\text { La ataguaya } \\
\text { (serpiente) }\end{array}$ & $\begin{array}{l}\text { Escogí a la ataguaya } \\
\text { porque es un animal } \\
\text { con bellos colores } \\
\text { (negro, blanco y rojo) } \\
\text { y es larga. }\end{array}$ & $\begin{array}{l}\text { venenosa y sus } \\
\text { colores son blanco, } \\
\text { rojo y negro. Su } \\
\text { mordida duele. }\end{array}$ & $\begin{array}{l}\text { Es importante porque } \\
\text { sin ella no se } \\
\text { reproducirían para } \\
\text { quabiese más } \\
\text { serpientes. }\end{array}$ \\
\hline
\end{tabular}

En cuanto al conocimiento que tanto niñas y niños tienen sobre estos animales, se puede clasificar en 5 categorías, las cuatro primeras dentro del conocimiento ecológico. En la Tabla No. 3 se hace una síntesis de lo explicitado por los niños y niñas de grado sexto.

Tabla. No 3. Aspectos señalados en cada categoría

\begin{tabular}{|c|c|}
\hline Categorías & $\begin{array}{l}\text { Aspectos señalados por los estudiantes con respecto a la } \\
\text { categoría que se identifica }\end{array}$ \\
\hline $\begin{array}{l}\text { Características } \\
\text { Físicas }\end{array}$ & $\begin{array}{l}\text { Describen, con menor o mayor grado de puntualidad, los animales de } \\
\text { la zona, haciendo referencia al tamaño, colores, señales particulares } \\
\text { en algunos, y la presencia de estructuras en otros. }\end{array}$ \\
\hline Hábitat & $\begin{array}{l}\text { Mencionan y puntualizan espacios donde los animales de su región } \\
\text { habitan y establecen relación con otras especies dentro de una } \\
\text { comunidad. Ej. Bosques, entre los árboles, entre las piedras, } \\
\text { chiqueros, ríos, nidos y cuevas, entre otros. Es decir que caracterizan } \\
\text { el nicho ecológico y la importancia que tiene los factores bióticos y } \\
\text { abióticos. }\end{array}$ \\
\hline Alimentación & $\begin{array}{l}\text { Identifican variados hábitos alimenticios de los animales que conocen. } \\
\text { Ejemplo. Caza ratones y sapos, come garrapatas. Pueden establecer } \\
\text { su ubicación en la cadena alimenticia como especies herbívoras, } \\
\text { carnívoras u omnívoras. }\end{array}$ \\
\hline Reproducción & $\begin{array}{l}\text { No solo la destacan sino que la señalan como parte importante de los } \\
\text { seres vivos, establecen su importancia para la preservación y } \\
\text { conservación. En algunos casos son específicos al decir que se } \\
\text { reproducen por huevos o son mamíferos. }\end{array}$ \\
\hline $\begin{array}{l}\text { Comportamiento y } \\
\text { funciones }\end{array}$ & $\begin{array}{l}\text { Son varios los aspectos que se señalan en esta categoría. Por } \\
\text { ejemplo, señalan que algunos animales son depredares, otros son } \\
\text { patógenos para el ser humano, y otros pueden ser parásitos. Todas } \\
\text { estas características permiten inferir sobre el conocimiento que los } \\
\text { niños y niñas tienen sobre el equilibrio de los ecosistemas. También } \\
\text { hacen referencia al comportamiento del animal y su papel en el } \\
\text { ecosistema. }\end{array}$ \\
\hline Usos & $\begin{array}{l}\text { En esta categoría podemos señalar el uso que los habitantes le dan a } \\
\text { algunos animales y la manera como se relacionan con ellos: } \\
\text { alimentación, mascotas y ornamentales como las aves. }\end{array}$ \\
\hline
\end{tabular}

En cuanto a los espacios de socialización, es importante anotar que cada actividad se compartió de diferentes maneras: exposición de los dibujos de los animales, presentación oral, dando respuesta a las preguntas de la Tabla No 2, de cada uno de los animales seleccionados, y finalmente, con la guía de la docente, se diligenció un formato 
Memorias del VIII Encuentro Nacional de Experiencias en Enseñanza de la Biología y la Educación Ambiental. III Congreso Nacional de Investigación en Enseñanza de la Biología.

con la información de todos los animales para que los niños lo tuvieran en sus carpetas, pues este material sirvió de insumo para posteriores actividades.

Se destaca la posibilidad que se abrió en todas las actividades de potenciar las diferentes manifestaciones del lenguaje: el habla espontánea, donde la opinión y los procesos de descripción, síntesis y argumentación fueron determinantes; el dibujo como una manera de representar sus saberes, y la escritura, aquella que permite comunicar a otros, pero también dejar huella de los saberes locales, en este caso. Este espacio también hizo posible compartir saberes, experiencias, intercambiar puntos de vista y procesos de negociación de significados.

\section{Conclusiones}

A continuación se puntualizan algunos aspectos que se desprenden del trabajo realizado, algunos de ellos merecen ser analizados y estudiados con mayor profundidad, mientras que otros pueden tomarse como invitaciones a repensar la enseñanza de las ciencias naturales considerando perspectivas y enfoques que tengan en cuenta el contexto cultural. Tal y como lo señala Hernández (2014), estos aspectos deben ser reflexionados y analizados no solo por quienes están a cargo de las políticas públicas del país, sino también, y quizás más importante aún, por las instituciones escolares y más concretamente, para el caso que no ocupa, del docente del sector rural, quien puede ser el gestor de nuevas formas de concebir el aprendizaje y la enseñanza de las ciencias naturales.

En acuerdo con Pardo y Gómez (2003), los conocimientos tradicionales que tienen las comunidades no marcadas étnicamente también deben interpretarse no solo como parte de la cultura sino como patrimonio natural y social. Por ello este tipo de trabajos abren espacios de reflexión y de identificación y reconocimiento de la riqueza natural, en este caso de la fauna de la región de Chingaza. Los saberes locales que tienen los estudiantes sobre los animales, y otros seres vivos, invitan a considerar una alternativa para crear puentes entre este tipo de conocimientos y los que propende, de manera general, la escuela actual en las ciencias naturales, muchas veces descontextualizados y desconectados de las realidades naturales y sociales de los estudiantes.

Finalmente, la identificación y caracterización del conocimiento tradicional sobre la naturaleza, enmarcado dentro de un tipo de conocimiento no es suficiente, lo importante es reconocerlo, lo cual significa establecer su importancia y poderlo vincular como insumo fundamental en el desarrollo de las clases de ciencias. Este aspecto demanda retos importantes para el docente, uno de ellos tiene que ver con las actividades de clase, las cuales, tal y como anotan Mosquera y Molina (2011) deben ser diseñadas a 
Bio - grafía. Escritos sobre la Biología y su Enseñanza. ISSN 2027-1034

Edición Extraordinaria. p.p. 148 - 156

Memorias del VIII Encuentro Nacional de Experiencias en Enseñanza de la Biología y la

Educación Ambiental. III Congreso Nacional de Investigación en Enseñanza de la Biología.

partir de reflexiones críticas, conscientes y analíticas del currículo. Lo anterior invita, como se dijo antes, a repensar y reconfigurar modelos alternativos de enseñanza de las ciencias más incluyentes y contextuales.

\section{Bibliografía}

Bolívar, A. (2004). Ciudadanía y Escuela Pública en el Contexto de Diversidad Cultural. Revista Mexicana de Investigación Educativa. 9, 9, 15-38.

Geertz, C. (1989). La interpretación de las culturas. Barcelona, España: Gedisa.

Maxwell, J. A. (1998). "Designing a Qualitative Study". En L. Bickman D. J. y Rog (Eds.), Handbook of Applied Social Research Method (p. 69-100), Thousand Oaks, CA, Sage.

Hernández, R (2013). Contexto cultural y currículum en la enseñanza de las ciencias. En: Enseñanza de las ciencias y cultura: múltiples aproximaciones. Fondo de publicaciones Universidad Distrital Francisco José de Caldas. Capítulo 7. 145-163.

McMillan, J y Schumacher, S. (2010). Investigación Educativa. Capítulo 10. Investigación con estudios de casos.

Molina, A., Mojica, L., López, D. (2005). Ideas de los niños y niñas sobre la naturaleza: estudio comparado. Colombia Revista Científica, 7, 41 - 62.

Molina, A.; Martínez, C. A.; Mosquera, C. J. y Mojica, L. (2009). Diversidad cultural $e$ implicaciones en la enseñanza de las ciencias: reflexiones y avances. En: Revista Colombiana de Educación, 56, pp. 103-128.

Molina, A y Uteges, G. (2011). Diversidad cultural, concepciones de los profesores y los ámbitos de sus prácticas. Dos estudios de caso. Revista de enseñanza de la física. 24, 7-26.

Pardo, M y Gómez, E. (2003). Etnobotánica: aprovechamiento tradicional de plantas y patrimonio cultural. Anales Jardín Botánico de Madrid, 60 (1).

Quenceno, R y Castaño, C. (2002). Introducción a la metodología de investigación cualitativa. Revista de Psicodidáctica. No 14. Pp. 1-27. 
Bio - grafía. Escritos sobre la Biología y su Enseñanza. ISSN 2027-1034

Edición Extraordinaria. p.p. $148-156$

Memorias del VIII Encuentro Nacional de Experiencias en Enseñanza de la Biología y la

Educación Ambiental. III Congreso Nacional de Investigación en Enseñanza de la Biología.

Vasilachis De Gialdano, I. (2006). Estrategias de investigación cualitativa. Barcelona: Gedisa. 